\title{
Influence of Flash Lamp Annealing on the Optical Properties of CIGS Layer
}

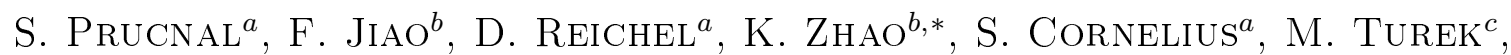 \\ K. Pyszniak ${ }^{c}$, A. Drozdziel ${ }^{c}$, W. Skorupa ${ }^{a}$, M. Helm ${ }^{a}$ And S. Zhou ${ }^{a}$ \\ ${ }^{a}$ Institute of Ion Beam Physics and Materials Research, Helmholtz-Zentrum Dresden-Rossendorf (HZDR) \\ P.O. Box 510119, Dresden 01314, Germany \\ ${ }^{b}$ State Key Laboratory of Nuclear Physics and Technology, School of Physics, Peking University \\ Beijing 100871, China \\ ${ }^{c}$ Institute of Physics, Maria Curie Skłodowska University, 20-031 Lublin, Poland

\begin{abstract}
Copper indium gallium diselenide (CIGS) becomes more significant for solar cell applications as an alternative to silicon. The quality of the layer has a critical impact on the final efficiency of the solar cell. An influence of the post-deposition millisecond range flash lamp annealing on the optical and microstructural properties of the CIGS films was investigated. Based on the Raman and photoluminescence spectroscopy, it is shown that flash lamp annealing reduces the defect concentration and leads to an increase of the photoluminescence intensity by a factor of six compared to the nonannealed sample. Moreover, after flash lamp annealing the degradation of the photoluminescence is significantly suppressed and the absolute absorption in the wavelength range of 200-1200 nm increases by $25 \%$.
\end{abstract}

DOI: $10.12693 /$ APhysPolA.125.1404

PACS: 88.40.jn, 61.72.Cc, 78.55.Hx

\section{Introduction}

Thin-film semiconductors have attracted much attention as an inexpensive and highly efficient solar cell. The most investigated thin-film materials for photovoltaic applications are different polycrystalline chalcopyrites $\left(\mathrm{A}^{\mathrm{I}} \mathrm{B}^{\mathrm{III}} \mathrm{C}_{2}^{\mathrm{VI}}\right)$. The copper indium gallium diselenide (CIGS) thin film solar cells deposited on glass reach efficiencies of up to $20.0 \%$ on the laboratory scale and $13.5 \%$ on large area which is comparable with polycrystalline silicon solar cells [1]. The recent world record for the energy conversion efficiency of flexible solar cells made of CIGS presented by the Swiss Federal Laboratories for Materials Science and Technology is 18.7\% [2]. Such high efficiency has been not obtained for any type of flexible solar cell grown on polymer or metal foil before. The main advantage of CIGS over different solar cell materials is its energy band gap tunability by varying layer composition during deposition. Using a non-uniform $\mathrm{Ga} / \mathrm{In}$ ratio throughout the film thickness the band gap can be adjusted over a range of $1.04 \mathrm{eV}$ for low gallium content up to $1.67 \mathrm{eV}$ for a gallium concentration higher than $70 \%[3,4]$. The band gap energy of CIGS determines directly the open circuit voltage $\left(V_{\mathrm{OC}}\right)$ and the efficiency of solar cell. Usually, the high-efficiency devices have minimum band gaps of 1.1-1.2 $\mathrm{eV}$ with the $V_{\mathrm{OC}}$ in the range of $650 \mathrm{mV}$. In fact, an increase of Ga content in CIGS films increases the $V_{\mathrm{OC}}$ but the efficiency

*corresponding author; e-mail: kzhao@pku.edu.cn drops down for Ga concentrations higher than $30 \%$ due to deep defect formation which are the non-radiative recombination centres $[5,6]$. Therefore, an improvement of the optical properties of the CIGS absorber is a milestone for high efficient solar cells production.

In this paper we have investigated the influence of post-deposition millisecond range flash lamp annealing (FLA) on the optical and microstructural properties of the CIGS layers deposited on glass. It is shown that after FLA the band gap photoluminescence intensity increases by a factor of six and the ordered defect compound (ODC) related peak in the Raman spectra disappears. Moreover, the main Raman peak observed from crystalline CIGS becomes narrower confirming the crystallinity improvement of CIGS films. An advantage of the FLA over conventional annealing techniques (furnace annealing, rapid thermal annealing) is that within milliseconds only the CIGS film is effectively heated to the required temperature without affecting the glass substrate. The FLA systems are successfully used for silicon recrystallization in many laboratories for applications ranging from ultra-shallow junction formation via recrystallization of amorphous layers to silicon based light emitter fabrication [7-9] and nanodot formation [10-12]. Recently our FLA system was applied for the solar cell emitter formation in multicrystalline solar grade $p$-type silicon wafers implanted by phosphorus [13].

\section{Experimental}

The CIGS films were deposited on molybdenum coated glass substrate. First, the metallic precursor $\mathrm{Cu}-\mathrm{In}-\mathrm{Ga}$ was deposited by DC sputtering. Copper and gallium 
were prepared by a $\mathrm{CuGa}$ alloy target $(30 \% \mathrm{Ga})$ and indium by a metalic In target. The sequence was $\mathrm{CuGa} / \mathrm{In} /$ $\mathrm{CuGa} / \mathrm{In}$. The substrate was kept at room temperature during deposition. Then the element Se was evaporated by solid particles. After deposition, the sample was pre-annealed at $400{ }^{\circ} \mathrm{C}$ in $\mathrm{Ar}$ atmosphere with a pressure of $200 \mathrm{~Pa}$ for $30 \mathrm{~min}$. The final thickness of the film is around $1 \mu \mathrm{m}$. After deposition and selenization samples were annealed by the flash lamp system for $20 \mathrm{~ms}$ in forming gas ambient $\left(\mathrm{N}_{95 \%}: \mathrm{H}_{5 \%}\right)$. For the optical and microstructural investigation of deposited and annealed samples $\mu$-Raman spectroscopy, photoluminescence, reflectivity and X-ray diffraction (XRD) were used. XRD was performed using a Siemens D5005 diffractometer with a $\mathrm{Cu}$-target source. The setup was equipped with a Göbel mirror to enhance the brilliance. The $\mu$-Raman and photoluminescence spectroscopy were performed under $532 \mathrm{~nm}$ Nd:YAG laser excitation. The room temperature PL spectra were recorded with a liquid nitrogen cooled InGaAs detector. For the reflectivity measurements the Solidspec-3700 DUV spectrophotometer in the wavelength range of 200-2000 $\mathrm{nm}$ was used.

\section{Results and discussion}

The quaternary chalcopyrite I-III-VI system with space group $I 42 d$ contains 8 atoms per primitive unit cell with 20 Raman active optical phonon modes $[14,15]$. In case of CIGS material, the dominant $A_{1}$ mode located at around $176 \pm 5 \mathrm{~cm}^{-1}$ (depends on the gallium content) represents the vibration of the selenium anions in the $x-y$ plane with other cations [16]. In fact, the position of the $A_{1}$ mode depends not only on the Ga concentration. Witte et al. have investigated an influence of the copper content on the optical and microstructural properties of CIGS absorbers [15]. They have observed a decrease of the $A_{1}$ mode frequency with increasing $\mathrm{Cu}$ content in CIGS films.

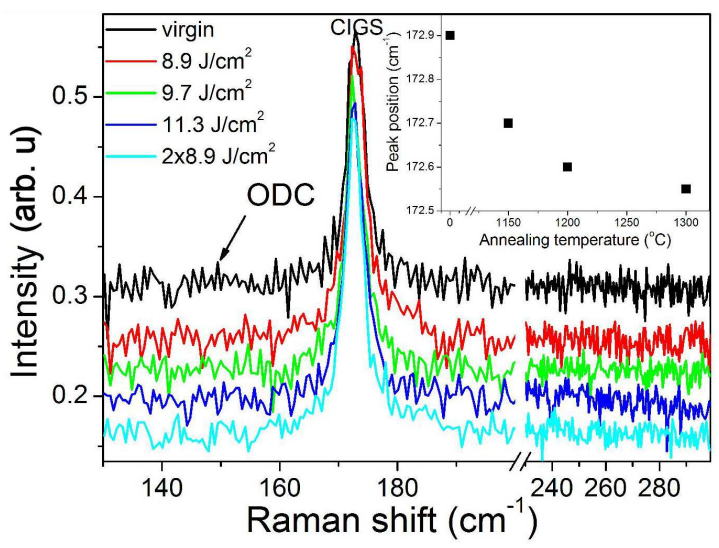

Fig. 1. $\mu$-Raman spectra of virgin and flash lamp annealed samples for $20 \mathrm{~ms}$. Inset shows the change of the maximum peak position ( $A_{1}$ mode) after flash lamp annealing. The spectra have been shifted vertically for clarity.
Figure 1 shows the $\mu$-Raman spectra obtained from samples after selenization and flash lamp annealing for $20 \mathrm{~ms}$ at different pulse energies in forming gas ambient. The main $A_{1}$ mode recorded from virgin sample (without FLA) is located at $172.9 \mathrm{~cm}^{-1}$ with FWHM of $3.52 \mathrm{~cm}^{-1}$. Besides the $A_{1}$ mode a weak broad band at $150 \mathrm{~cm}^{-1}$ is visible. This band corresponds to the ODC commonly present in $\mathrm{Cu}$-poor CIGS absorber or low-quality chalcopyrite crystals. Simultaneously the high quality of the CIGS absorber is confirmed by absence of the CuSe or $\mathrm{Cu}_{2}$ Se Raman mode at around $260 \mathrm{~cm}^{-1}$ [17].

After flash lamp annealing the ODC related band disappears and a slight down shift of the $A_{1}$ mode was observed (see inset, Fig. 1). Moreover, the FWHM of $A_{1}$ mode decreases from $3.52 \mathrm{~cm}^{-1}$ for virgin down to $3.37 \mathrm{~cm}^{-1}$ for double flashed sample $\left(2 \times 9.7 \mathrm{~J} / \mathrm{cm}^{2}\right)$ and the relative intensity of the $A_{1}$ mode peak increases. Taking into account that the composition of the investigated samples was the same in all experiments, the vanishing of the ODC mode and shift of the $A_{1}$ mode in the Raman spectra to the lower frequency after post-selenization annealing indicates an improvement of the CIGS absorber quality due to the incorporation of free copper atoms into the chalcopyrite system during flash lamp annealing.

In general, the optical properties of any photovoltaic material determine finally the efficiency of solar cell devices. Shirakata et al. have investigated the correlation between photoluminescence intensity and conversion efficiency $(\eta)$ in CIGS films with emphasis on the near-band-edge photoluminescence (NBE-PL) of CIGS during the solar cell fabrication process [18]. The highest $\eta$ $(9.1 \%)$ was obtained from samples revealing the highest near-band-edge emission [15]. Moreover, strong degradation of the PL efficiency with time was observed. In case of non-passivated surface of the CIGS absorber the NBE-PL intensity decreases by two orders of magnitude within $24 \mathrm{~h}$. The exact mechanism of PL quenching is unknown [19]. One explanation is the enhancement of the surface recombination process by some oxide formation on the surface [20].

Figure 2a shows the room-temperature photoluminescence spectra obtained from virgin and flash lamp annealed samples. The PL spectrum of the virgin sample consists of broad band with a maximum intensity located at $1210 \mathrm{~nm}$ due to near-band-edge emission and free-to-bound (FB) transition at about $1250 \mathrm{~nm} \mathrm{[21,} \mathrm{22].} \mathrm{The}$ flash lamp annealing leads to an enhancement of the maximum NBE-PL intensity and shifts its position of about $10 \mathrm{~nm}$ to the longer wavelength (smaller band gap) and the separation between the two observed emissions (NBE and FB) is clearly visible. Typically, the band gap energy of CIGS samples is in the range of $0.98-1.7 \mathrm{eV}$ depending on the gallium content [4].

Based on the PL investigation the band gap of our samples is $1.02 \pm 0.01 \mathrm{eV}$ which is in good agreement with literature for the CIGS absorber containing less than $20 \%$ of gallium. The small shift of the maximum PL peak intensity after FLA $(0.01 \mathrm{eV})$ can be due to free copper 

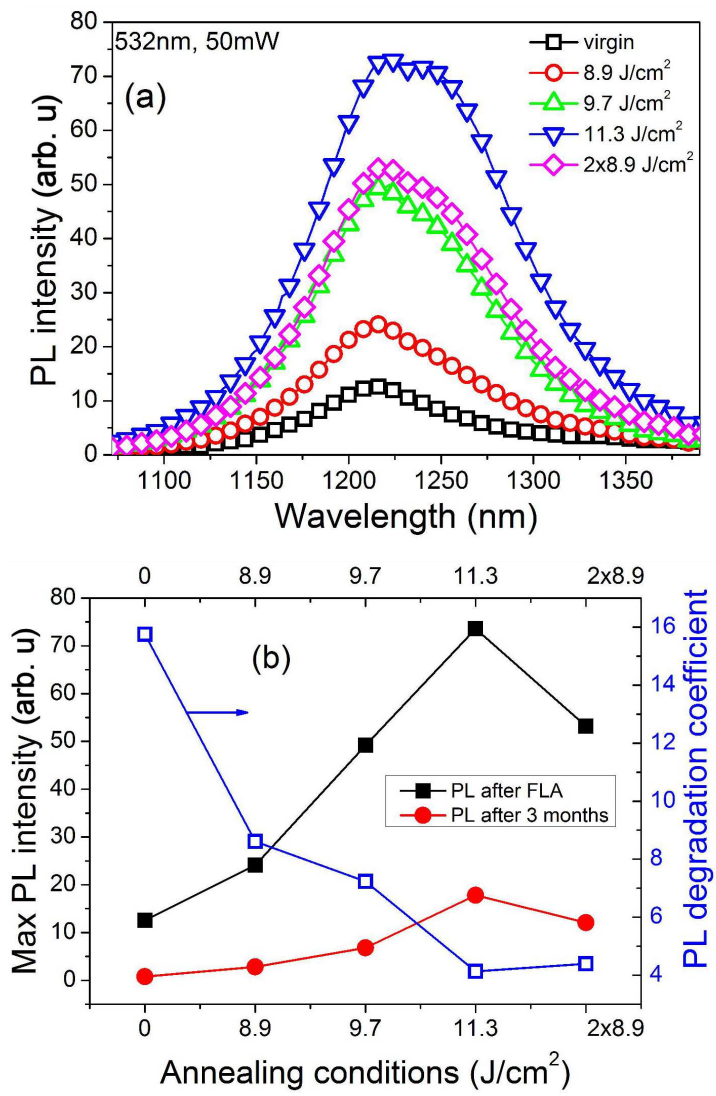

Fig. 2. Room temperature photoluminescence intensity obtained from virgin and flash lamp annealed samples under $532 \mathrm{~nm}$ laser excitation just after preparation (a) and the change of the maximum PL intensity after 3 months (b).

atoms incorporated into the chalcopyrite system. Our assumption correlates with Raman spectra where after annealing the ODC related peak vanishes completely. For samples annealed at $11.3 \mathrm{~J} / \mathrm{cm}^{2}$ for $20 \mathrm{~ms}$ the NBE-PL intensity increases by a factor of six compared to the virgin sample.

Figure $2 \mathrm{~b}$ shows the change of the maximum NBE-PL intensity obtained from samples after annealing and three months later (left scale) and NBE-PL degradation factor within the same time (right scale). In case of virgin sample the NBE-PL after three months decreases by factor of 16 while the flashed sample reveals to be much more stable. After flash lamp annealing in the forming gas atmosphere $(95 \% \mathrm{~N}+5 \% \mathrm{H})$ the degradation of NBE-PL is reduced by factor of two for $8.9 \mathrm{~J} / \mathrm{cm}^{2}$ and by factor of four for $11.3 \mathrm{~J} / \mathrm{cm}^{2}$ flash. Annealing at energy higher than $11.3 \mathrm{~J} / \mathrm{cm}^{2}$ leads to the decomposition of the CIGS absorber. An influence of the annealing conditions under different atmospheres on the electronic properties of the CIGS layer was investigated by Sakurai et al. [23]. They have found that an annealing of CIGS films in vacuum or oxygen ambient promotes selenium vacancy formation and indium oxidation at the surface which degrades the electronic properties of the film, although such degradation was not observed after annealing in nitrogen.

Hydrogen introduced to CIGS absorber neutralizes negatively charged copper vacancies, reduces the indium oxide, enhances in-diffusion of indium and may activate selenium vacancy formation at the surface [24]. Hence, the post-selenization annealing at forming gas atmosphere reveals to be the most suitable for high-efficiency CIGS absorber solar cell formation. Both nitrogen and hydrogen passivate efficiently the surface of the CIGS absorber and supress the oxidation which finally reduces the degradation of the optical properties of CIGS films.

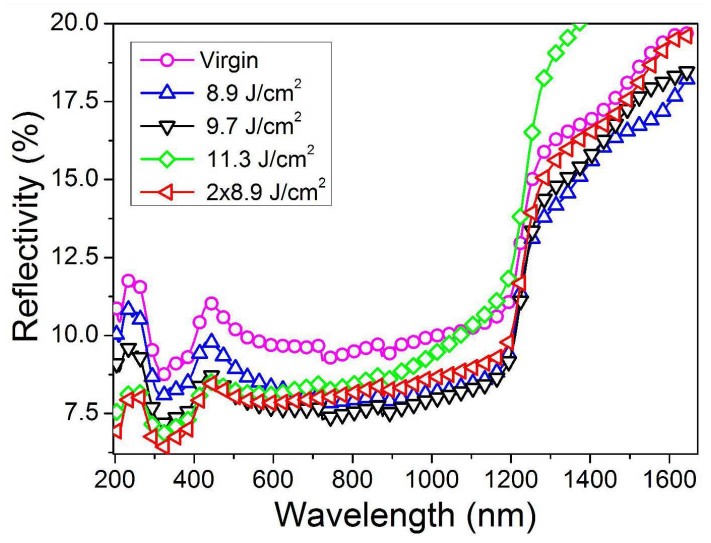

Fig. 3. Reflectivity spectra of virgin and flash lamp annealed samples as function of incident wavelength.

An improvement of the CIGS absorber quality after FLA annealing was observed by the reflectivity measurements as well. Figure 3 shows the reflectivity spectra of virgin and FLA annealed samples as function of incident light measured in the wavelength range of 200-1600 nm. The average reflectivity measured in the range of 300 $1050 \mathrm{~nm}$ decrease from $10 \%$ for virgin sample to $7.9 \%$ for sample annealed at $9.7 \mathrm{~J} / \mathrm{cm}^{2}$. It corresponds to an increase of the absolute absorption by $25 \%$. An increase of the reflectivity above $1200 \mathrm{~nm}$ is due to incident light reflection from the back molybdenum layer. The band gap energy of CIGS absorber evaluated from the reflectivity measurements is in the range of $1 \mathrm{eV}$ which is in good agreement with that obtained by photoluminescence and $\mu$-Raman spectroscopy.

The quality of the crystal structure of the chalcopyrite CIGS system was investigated by means of XRD. Figure 4 shows the XRD patterns of CIGS films grown on molybdenum coated glass substrate. As can be seen, the XRD spectra consist of sharp peaks relating to crystalline CIGS and the molybdenum layer (Mo (110)). The main XRD peak at $26.7(2 \theta)$ corresponds to (112) orientation of CIGS and it is three times higher than second intense $(220 / 204)$ peak [25]. The intensity ratio of the main (112) and $(220 / 204)$ peaks remain unchanged after flash lamp annealing. The peak position and FWHM of all peaks seems to be unaffected as well. Hence we can conclude that after deposition and selenization (virgin sample) the 


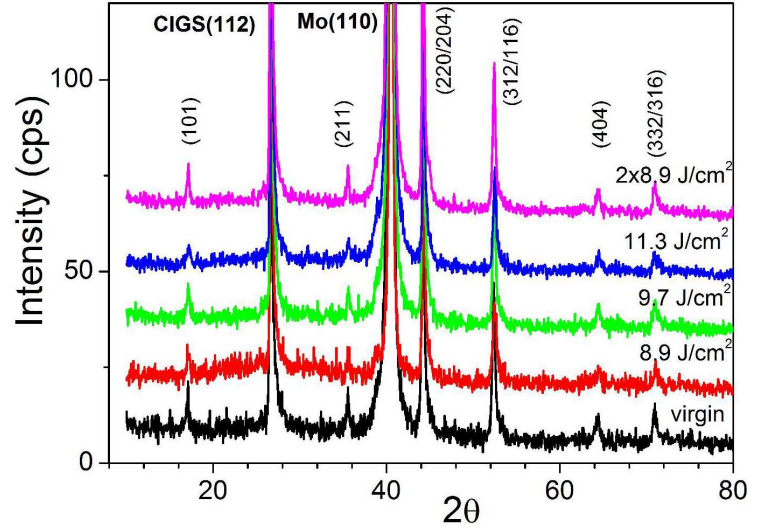

Fig. 4. XRD spectra obtained from virgin and flash lamp annealed samples. The spectra have been shifted vertically for clarity.

CIGS absorber is a high quality (112) preferentially oriented polycrystalline one and the post-selenization FLA did not affect the crystal quality but improves the optical properties by surface passivation and defect concentration reduction.

\section{Conclusions}

The influence of the post-selenization thermal treatment on the optical and microstructural properties of CIGS absorbers was investigated. It is shown that the millisecond range flash lamp annealing improves significantly the optical properties of the CIGS films. The PL intensity increases by a factor of six and the ODC defects are removed. Simultaneously the crystal structure is not affected. Due to the surface passivation during FLA in forming gas the CIGS layer becomes more stable against oxidation. Moreover, the FLA treatment improves the absolute absorption by $25 \%$.

\section{Acknowledgments}

The work was partially supported by the Helmholtz-Gemeinschaft Deutscher Forschungszentren (HGF-VH-NG-713).

\section{References}

[1] M. Bär, I. Repins, M.A. Contreras, L. Weinhardt, R. Noufi, C. Heske, Appl. Phys. Lett. 95, 052106 (2009).

[2] www.solarbuzz.com/node/2573.

[3] M. Gloeckler, J.R. Sites, J. Phys. Chem. Solids 66, 1891 (2005).

[4] A. Morales-Acevedo, Energy Procedia 2, 169 (2010).

[5] G. Hanna, A. Jasenek, U. Rau, H.W. Schock, Thin Solid Films 387, 71 (2001).

[6] W.N. Shafarman, R. Klenk, B.E. McCandless, J. Appl. Phys. 79, 7324 (1996).
[7] W. Skorupa, T. Gebel, R.A. Yankov, S. Paul, W. Lerch, D.F. Downey, E.A. Arevalo, J. Electrochem. Soc. 152, G436 (2005).

[8] F. Terai, S. Matunaka, A. Tauchi, Ch. Ichimura. T. Nagatomo, T. Homma, J. Electrochem. Soc. 153 , H147 (2006).

[9] S. Prucnal, J.M. Sun, A. Muecklich, W. Skorupa, Electrochem. Solid-State Lett. 10, H50 (2007).

[10] S. Prucnal, M. Turek, A. Drozdziel, K. Pyszniak, S.Q. Zhou, A. Kanjilal, W. Skorupa, J. Zuk, Appl. Phys. B 101, 315 (2010).

[11] S. Prucnal, M. Turek, A. Drozdziel, K. Pyszniak, A. Wójtowicz, S.Q. Zhou, A. Kanjilal, A. Shalimov, W. Skorupa, J. Zuk, Cent. Eur. J. Phys. 9, 338 (2011).

[12] S. Prucnal, S.-Q. Zhou, X. Ou, H. Reuther, M.O. Liedke, A. Mücklich, M. Helm, J. Zuk, M. Turek, K. Pyszniak, W. Skorupa, Nanotechnology 23, 485204 (2012).

[13] S. Prucnal, B. Abendroth, K. Krockert, K. König, D. Henke, A. Kolitsch, H.J. Möller, W. Skorupa, J. Appl. Phys. 111, 123104 (2012).

[14] J. Łażewski, H. Neumann, K. Parlinski, G. Lippold, J. Stanbery, Phys Rev. B 68, 144108 (2003).

[15] W. Witte, R. Kniese, M. Powalla, Thin Solid Films 517, 867 (2008).

[16] H. Tanino, T. Maeda, H. Fujikake, H. Nakanishi, S. Endo, T. Irie, Phys. Rev. B 45, 13323 (1992).

[17] D. Papadimitriou, N. Esser, C. Xue, Phys. Status Solidi B 242, 2633 (2005).

[18] S. Shirakata, S. Yudate, J. Honda, N. Iwado, Jpn. J. Appl. Phys. 50, 05FC02 (2011).

[19] W.K. Metzger, I.L. Repins, M.A. Contreras, Appl. Phys. Lett. 93, 022110 (2008).

[20] S. Kijima, T. Nakada, Appl. Phys. Express 1, 075002 (2008).

[21] S. Shirakata, T. Nakada, Phys. Status Solidi C 6 , 1059 (2009).

[22] S. Niki, Y. Makita, A. Yamada, A. Obara, S. Misawa, O. Igarashi, K. Aoki, N. Kutsuwada, Jpn. J. Appl. Phys. 33, L500 (1994).

[23] T. Sakurai, N. Ishida, S. Ishizuka, M.M. Islam, A. Kasai, K. Matsubara, K. Sakurai, A. Yamada, K. Akimoto, S. Niki, Thin Solid Films 516, 7036 (2008).

[24] K. Otte, T. Chasse, G. Lippold, B. Rauschenbach, R. Szargan, J. Appl. Phys. 91, 1624 (2002).

[25] T. Mise, T. Nakada, Solar Energy Mater. Solar Cells 93, 1000 (2009). 